\title{
Plasma levels of tumour necrosis factor- $\alpha$ and adiponectin can differentiate patients with psoriatic arthritis from those with psoriasis
}

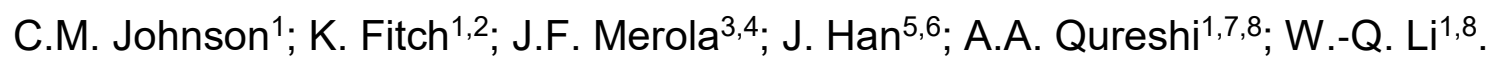

1. Department of Dermatology, Warren Alpert Medical School, Brown University, Providence, RI, U.S.A.

2. Warren Alpert Medical School, Brown University, Providence, RI, U.S.A.

3. Department of Dermatology, Brigham and Women's Hospital, Harvard Medical School, Boston, MA, U.S.A.

4. Division of Rheumatology, Department of Medicine, Brigham and Women's Hospital, Harvard Medical School, Boston, MA, U.S.A.

5. Department of Epidemiology, Richard M. Fairbanks School of Public Health, Indiana University, Indianapolis, IN, U.S.A.

6. Melvin and Bren Simon Cancer Center, Indiana University, Indianapolis, IN, U.S.A.

7. Department of Dermatology, Rhode Island Hospital, Providence, RI, U.S.A.

8. Department of Epidemiology, School of Public Health, Brown University, Providence, RI, U.S.A.

This is the author's manuscript of the article published in final edited form as:

Johnson, C. M., Fitch, K., Merola, J. F., Han, J., Qureshi, A. A., \& Li, W.-Q. (2019). Plasma levels of tumour necrosis factor- $\alpha$ and adiponectin can differentiate patients with psoriatic arthritis from those with psoriasis. British Journal of Dermatology, 181(2), 379-380. https://doi.org/10.1111/bjd.17700 
Dear Editor, Psoriasis (PsO) and psoriatic arthritis (PsA) are inflammatory disorders. Circulating biomarkers of inflammation such as interleukin (IL)-6, tumour necrosis factor (TNF)- $\alpha$, and C-reactive protein (CRP) have been associated with disease severity and progression of PsO and PsA.1 Adiponectin and leptin are adipose-derived cytokines, recognized as key regulators of body weight and metabolism. 2 Whether circulating levels of these inflammatory and metabolic biomarkers may predict the risk of PsA in patients with $\mathrm{PsO}$ remains unclear.

We included 180 patients with PsO only and 143 patients with an additional diagnosis of PsA from the Psoriatic Arthritis and Psoriasis Follow-up Study, a multicentre registry at Brigham and Women's Hospital, U.S.A. Participant consent was obtained; the study was approved by the institutional review board of Brigham and Women's hospital [protocol number: 2012-P-001325(1)].

Patients with PsO and those with PsA were similar in age (median 51 years, interquartile range 40-62). Patients with PsA included a higher proportion of men (56.2\%) than those with $\mathrm{PsO}(50.0 \%)$. Plasma levels of IL-6, CRP, TNF- $\alpha$, leptin, total adiponectin, and high molecular-weight (HMW) adiponectin were assessed by ultrasensitive enzyme-linked immunosorbent assay or immunoturbidimetric assay. Median plasma levels of TNF- $\alpha$ were significantly higher in patients with PsA compared with those with PsO (3.27 vs. $\left.1.32 \mathrm{pg} \mathrm{mL}^{-1}, P<0.001\right)$. Furthermore, there was a slight decrease among patients with PsA in median total adiponectin (4.66 vs. $\left.5.36 \mu \mathrm{gL}^{-1}, P=0.15\right)$ and $\mathrm{HMW}$ adiponectin (2.58 vs. $\left.3.01 \mu \mathrm{gL}^{-1}, P=0 \cdot 12\right)$.

Logistic regression analyses were conducted to calculate the odds ratios (ORs) and $95 \%$ confidence intervals ( $\mathrm{Cls}$ ) for associations between plasma biomarkers and PsA, compared with $\mathrm{PsO}$. Using median plasma levels of each biomarker in the $\mathrm{PsO}$ group as the cut-off, patients were separated into high and low subsets. A high TNF- $\alpha$ level was associated with increased odds of PsA (multivariate adjusted OR $=2 \cdot 25,95 \% \mathrm{Cl} 1 \cdot 41-$ 3.61). A significantly inverse association was found between high total adiponectin and odds of PsA (multivariate adjusted $\mathrm{OR}=0.61,95 \% \mathrm{Cl} 0.39-0.96$ ). An inverse association was also found for HMW adiponectin with PsA, although the association became 
marginally significant after multivariate adjustment $(\mathrm{OR}=0.64,95 \% \mathrm{Cl} 0 \cdot 41-1 \cdot 01)$ (Table 1).

Table 1. The association between biomarker plasma level and odds ratio (OR) of psoriatic arthritis

\begin{tabular}{|c|c|c|c|c|}
\hline & $\begin{array}{l}\text { Psoriasis } \\
\mathrm{n}(\%)\end{array}$ & $\begin{array}{l}\text { Psoriatic arthritis } \\
\mathrm{n}(\%)\end{array}$ & OR $(95 \% \mathrm{CI})$ & $\mathrm{OR}^{\mathrm{a}}(95 \% \mathrm{CI})$ \\
\hline \multicolumn{5}{|c|}{ Interleukin-6 $\left(\mathrm{pg} \mathrm{mL}{ }^{-1}\right)^{\mathrm{b}}$} \\
\hline$<1.94$ & $90(50 \cdot 0)$ & $66(46 \cdot 2)$ & 1.00 & 1.00 \\
\hline$\geq 1.94$ & $90(50 \cdot 0)$ & $77(53.8)$ & $1.17(0.75-1.81)$ & $1.28(0.81-2.03)$ \\
\hline \multicolumn{5}{|c|}{ C-reactive protein $\left(\mathrm{mg} \mathrm{dL}^{-1}\right)^{\mathrm{b}}$} \\
\hline$<0.20$ & $87(48 \cdot 3)$ & $65(45 \cdot 5)$ & 1.00 & 1.00 \\
\hline$\geq 0.20$ & $93(51 \cdot 7)$ & $78(54 \cdot 5)$ & $1.12(0.72-1.74)$ & $1.24(0.79-1.95)$ \\
\hline \multicolumn{5}{|c|}{ Human leptin $\left(\mathrm{ng} \mathrm{mL}^{-1}\right)^{\mathrm{b}}$} \\
\hline$<10 \cdot 20$ & $89(49.4)$ & $66(46 \cdot 2)$ & 1.00 & 1.00 \\
\hline$\geq 10 \cdot 20$ & $91(50 \cdot 6)$ & $77(53 \cdot 8)$ & $1.14(0.74-1.77)$ & $1.21(0.77-1.90)$ \\
\hline \multicolumn{5}{|c|}{ Tumour necrosis factor- $\alpha\left(\mathrm{pg} \mathrm{mL}^{-1}\right)^{\mathrm{b}}$} \\
\hline$<1.32$ & $89(49.4)$ & $44(30.8)$ & 1.00 & 1.00 \\
\hline$\geq 1.32$ & $91(50 \cdot 6)$ & $99(69 \cdot 2)$ & $2.20(1.39-3.49)$ & $2.25(1.41-3.61)$ \\
\hline \multicolumn{5}{|c|}{ Adiponectin (total) $\left(\mu \mathrm{g} \mathrm{mL}^{-1}\right)^{\mathrm{b}}$} \\
\hline$<5.36$ & $90(50 \cdot 0)$ & $89(62 \cdot 2)$ & 1.00 & 1.00 \\
\hline$\geq 5.36$ & $90(50 \cdot 0)$ & $54(37 \cdot 8)$ & $0.61(0.39-0.95)$ & $0.61(0.39-0.96)$ \\
\hline \multicolumn{5}{|c|}{ Adiponectin (high molecular weight) ( $\left.\mu \mathrm{g} \mathrm{mL}^{-1}\right)^{\mathrm{b}}$} \\
\hline$<3.01$ & $90(50 \cdot 0)$ & $88(61 \cdot 5)$ & 1.00 & 1.00 \\
\hline$\geq 3.01$ & $90(50 \cdot 0)$ & $55(38 \cdot 5)$ & $0.63(0.40-0.98)$ & $0.64(0.41-1.01)$ \\
\hline
\end{tabular}

- $\mathrm{Cl}$, confidence interval. aAjusted for age, sex, body mass index, smoking and alcohol intake status. ${ }^{b}$ Categorical variables were dichotomized based on the median biomarker levels of patients with only psoriasis.

Previously, Chandran et al . identified serum CRP as a potential soluble marker in PsA vs. PsO only. $\underline{3}$ The potential prognostic value of soluble IL-6, adiponectin and leptin in differentiating PsA from PsO has also been suggested, but significantly elevated leptin was found only among female participants. $\underline{4}, \underline{5}$ Here we did not find a significant 
association between plasma CRP, IL-6, or leptin levels and PsA. However, we did note significantly increased concentrations of plasma TNF- $\alpha$ in PsA vs. PsO. Elevated TNF- $\alpha$ has been associated with metabolic dysfunction and increased body adiposity, while obesity may lead to low-grade systemic inflammation, and obese individuals have a high risk of PsO and PsA. $\underline{6}$ To our knowledge, no previous study has reported a significant difference in circulating TNF- $\alpha$ level between PsA and PsO.

Adiponectin increases insulin resistance and may play a role in attenuating inflammatory activity. Adiponectin can indirectly suppress production of TNF- $\alpha$, and has been suggested as a negative regulator of $\mathrm{PsO}$ progression. $2, \underline{7}$ Proinflammatory cytokines such as TNF- $\alpha$ and IL- 6 may decrease production - and thereby circulating levels - of adiponectin. $\underline{8}$ We found a significantly inverse association between plasma adiponectin level and PsA, consistent with the current understanding of the role of adiponectin in inflammatory disease processes.

We further examined whether smoking, alcohol intake and body mass index modified the associations of PsA with plasma biomarkers. We found an inverse association of PsA with total or HMW adiponectin only among participants reporting alcohol intake, demonstrating significant interactions $(P$-value for the interaction $=0.02$ for total adiponectin and 0.03 for HMW adiponectin). Further research is merited to better understand the implications of the interactions for PsA.

We acknowledge several limitations. Our study was based on a modest sample size in a cross-sectional setting, which would merely suggest the differentiation between PsA and $\mathrm{PsO}$ by serum biomarkers. We did not collect complete information on PsO and PsA treatment, and were not able to adjust for treatment-related factors. Additionally, we did not compare our findings with those for healthy individuals without psoriatic disease, so extrapolation of our results to other settings should be cautious.

In conclusion, high plasma TNF- $\alpha$ level was associated with increased odds of PsA, and adiponectin level was inversely associated with PsA compared with PsO. Furthermore, alcohol consumption significantly modified the inverse association between adiponectin and the odds of PsA. Further large-scale investigation in a prospective setting of patients 
with PsO would be warranted, if a clinically useful screening test is to be developed for risk prediction of PsA based on circulating biomarkers.

Funding sources: the study was supported by the Investigator Initiated Grant from Amgen, Inc., who were not involved in the data collection, data analysis, manuscript writing and publication.

Conflicts of interest: A.A.Q. serves as a consultant for AbbVie, Amgen, Centers for Disease Control and Prevention, Janssen, Merck, Novartis and Pfizer, with honoraria donated to charity. J.F.M. is a consultant and/or investigator for Biogen IDEC, AbbVie, Amgen, Eli Lilly, Novartis, Pfizer, Janssen, UCB, Samumed, Science 37, Celgene, Sanofi Regeneron, Merck and GSK. The other authors declare no conflicts of interest.

C.M.J and K.F. contributed equally to this paper.

\section{REFERENCES}

Villanova, F., Meglio, P. D., \& Nestle, F. O. (2013). Biomarkers in psoriasis and psoriatic arthritis. Annals of the Rheumatic Diseases, 72(suppl 2), ii104-ii110. https://doi.org/10.1136/annrheumdis-2012-203037

Shibata S, Tada Y, Hau CS et al . Adiponectin regulates psoriasiform skin inflammation by suppressing IL-17 production from $ү \delta-T$ cells. Nat Commun 2015; 6: 7687.

Chandran, V., Cook, R. J., Edwin, J., Shen, H., Pellett, F. J., Shanmugarajah, S., Rosen, C. F., \& Gladman, D. D. (2010). Soluble biomarkers differentiate patients with psoriatic arthritis from those with psoriasis without arthritis. Rheumatology, 49(7), 1399-1405. https://doi.org/10.1093/rheumatology/keq105

Alenius, G.-M., Eriksson, C., \& Rantapää Dahlqvist, S. (2009). Interleukin-6 and soluble interleukin-2 receptor alpha-markers of inflammation in patients with psoriatic arthritis? Clinical and Experimental Rheumatology, 27(1), 120-123.

Eder, L., Jayakar, J., Pollock, R., Pellett, F., Thavaneswaran, A., Chandran, V., Rosen, C. F., \& Gladman, D. D. (2013). Serum adipokines in patients with psoriatic arthritis and psoriasis alone and their correlation with disease activity. Annals of the Rheumatic Diseases, 72(12), 1956-1961. https://doi.org/10.1136/annrheumdis-2012-202325 
Cawthorn, W. P., \& Sethi, J. K. (2008). TNF- $\alpha$ and adipocyte biology. FEBS Letters, 582(1), 117-131. https://doi.org/10.1016/j.febslet.2007.11.051

Shibata, S., Tada, Y., Hau, C., Tatsuta, A., Yamamoto, M., Kamata, M., Karakawa, M., Asano, Y., Mitsui, H., Sugaya, M., Kadono, T., Saeki, H., Kanda, N., \& Sato, S. (2011). Adiponectin as an anti-inflammatory factor in the pathogenesis of psoriasis: Induction of elevated serum adiponectin levels following therapy. British Journal of Dermatology, 164(3), 667-670. https://doi.org/10.1111/j.1365-2133.2010.10123.x

Fantuzzi, G. (2008). Adiponectin and inflammation: Consensus and controversy. Journal of Allergy and Clinical Immunology, 121(2), 326-330. https://doi.org/10.1016/j.jaci.2007.10.018 